\title{
LA REHABILITATION DES INFRASTRUCTURES SPORTIVES (Étude de cas et diagnostic technique du stade de football Chahid Hamlaoui de Constantine)
}

\section{Résumé:}

Dans le cadre d'une stratégie locale de mise à niveau de toutes les infrastructures sportives de la wilaya de Constantine, une nouvelle approche aura pour but de redynamiser la pratique sportive et à promouvoir le sport dans cette wilaya.

Définir d'une part la situation actuelle des infrastructures de l'office de parc omnisports de la Wilaya de Constantine, d'autre part identifier les besoins en terme qualitatif, technique et sécuritaire des équipements.

Nous avons utilisé la méthode d'enquête qui était basée sur la récolte d'information, l'observation, et le diagnostic visuel de l'état du stade de football a été réaliser avec le chef de division technique, et l'architecte en particulier.

Cette opération de renforcement et de réhabilitation a fait l'impact sur la dynamisation des activités sportives. ce complexe sportif permettra à Constantine de postuler à l'accueil des manifestations sportives de haut niveau ; ainsi la promotion du sport d'élite notamment le football.

Mots clés : stade de football, diagnostique technique, travaux de réhabilitations, mesures de sécurités

في اطار استر اتيجية محلية قصد ترميم المر افق الرياضية من طرف ولاية قسنطينة لدفع تنمية و تطوير الرياضة تم تسطير عملية

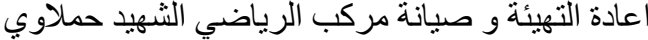

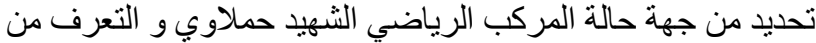
جهة اخرى احتياجات المركب من الناحية التقنية و الامنية للعتاد الرياضي التئي

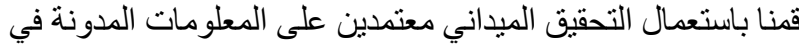

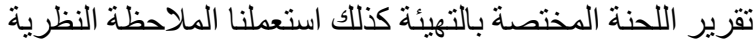

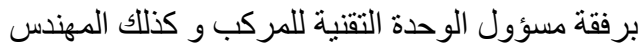

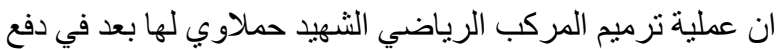

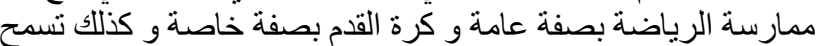

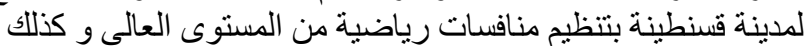
ترقية الرياضة و خاصنة كرة القدم

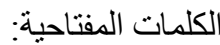
مقاييس الامن,عملية الترميم , تشخيص تقني ,ملعب كرة القدم

\section{Introduction :}

L'office de parc omnisports de la ville de Constantine et un établissement public à caractère administratif doté de la personnalité morale et de l'autonomie financière; de se fait il est soumis dans sa gestion des ressources humaines au régime de la fonction publique et financière aux règles générales de la comptabilité publique dont la trésorerie de wilaya et le comptable assignataire, comme elle est assignée au visa préalable du contrôle financier. 
Il est administré par un conseil d'administration et dirigé par un directeur conformément aux textes de création.

Par arrêté $\mathrm{N}^{\circ} 70.038$ du 05-05-1970 portant affectation d'un terrain d'une superficie de 49 hectares au profit du ministère de la jeunesse et des sports pour servir d'assiette à l'implantation d'un parc des sports à la ville de Constantine. (3)

La réalisation de cette infrastructure s'est effectué en plusieurs tranches suivantes une conception sociale à savoir du mûr symbolique terrain en tartan artificiel permettant la possibilité d'une forte participation sportive et fut inauguré en juin 1974.

$\mathrm{Ne}$ disposant pas de statut, l'infrastructure sportive est mise à la disposition de la direction de la jeunesse et des sports de la wilaya de Constantine, qui à pris la charge de gestion jusqu'à août 1977.

En effet un décret de création des offices des parcs omnisports des wilayas ( OPOW ) à été promulgué le 06-08-1977 portant : création, organisation et fonctionnement des offices des parcs omnisports des wilaya en définissant la nature juridique de cette infrastructure comme établissement publics à caractère administratif doté de la personnalité morale et de l'autonomie financière.(3)

\section{Composition de l'Infrastructure Sportive}

- stade de Football d'une capacité de 35000 places dont 15000 places couvertes terrain principale $(120 \mathrm{~m}-90 \mathrm{~m})$ en gazon naturel.

- piste d'athlétisme (08 couloires).

- 04 fosses de sable pour saut.

- 01 fosse pour steeple

- 12 vestiaires pour les joueurs.

- 02 vestiaires pour les arbitres.

- 11 douches collectives.

- 02 cabines de presse $\left(16 \mathrm{~m}^{2}\right)$.

- parking de capacité de 600 places.

Stade réplique (annexe) en gazon naturel de capacité de 3000 places

- 1 terrain réplique en gazon naturel

- 1 piste d'athlétisme.

- Gradin de capacité de 3000 places.

- parking de capacité de 200 places.

Centre d'hébergement et de restauration dite coupole $(\mathrm{R}+1)$ affecté par convention à l'association sportive MOC depuis 1996.

À l'extérieure un parking de capacité de 30 voitures.

L'administration

-Composé de 12 bureaux $\left(120 \mathrm{~m}^{2)}\right.$

-02 sanitaires.

-garage $\left(80 \mathrm{~m}^{2}\right)$ scindé en 03 parties (service atelier technique -foyer-atelier de peinture). 
-parking d'administration de capacité de 40 voitures.

-la Division Administrative et Financière : Chargée de la gestion des moyens humains matériels et financiers de l'office de parcs omnisports.

-la Division Technique: Chargée d'assurer les travaux d'entretien de réparation et d'aménagement des installations sportives de l'office.

\section{1. Problématique :}

Les équipements sportifs sont dans leurs immenses majorités sont des équipements publics, toutefois qu'ils aient vocation à remplir une mission d'intérêt général ou à poursuivre un but commercial, en outre ils doivent être soumis aux normes spécifiques en matière technique et sécuritaire.

Est-ce que le stade Hamlaoui de Constantine offre un service de qualité adaptés aux attentes de leur usagers, soient des pratiquants ou spectateurs?

2. 2. Objectifs : Les objectifs de cet article est:

- définir la situation actuelle du stade Chahid Hamlaoui de la ville de Constantine

- identifié les besoins en terme qualitatifs, techniques et sécuritaires des ces équipements.

\section{3. Hypothèses :}

Les critères techniques des équipements analysés du stade de football Chahid Hamlaoui ne sont pas conformes aux exigences de sécurité.

Ce défaut pourrait être dû au manque d'une politique de maintenance pertinente au sein de cet établissement sportif.

2.4. Méthodes : -Un état de lieux, et une identification de la situation de l'établissement sur le plan sécuritaire.

Nous avons utilisé la méthode d'enquête qui était basée sur la récolte d'information, l'observation, et le diagnostic visuel sur l'état technique et fonctionnel des équipements du stade avec le chef de division technique, et l'architecte en particulier.

\section{Discussion de l'état et diagnostic du stade principal :}

- Salle de contrôle: Chaque stade doit être doté d'une salle de contrôle ayant une vue générale de tout l'intérieur du stade et étant équipée d'un système de haut-parleurs et de moniteurs de vidéosurveillance. Les caméras de surveillance offrant les fonctions de prise de vue panoramique, assurant une surveillance des entrées des spectateurs, ainsi que tout le secteur du stade.

L'état actuel : Un local réservé aux responsables de la sécurité, à savoir l'officier de police responsable du match, le responsable de la sécurité du stade et leurs personnels. (2) 
-Local du délégué: local réservé au délégué de match de l'UEFA et à l'observateur d'arbitres.

-Zone pour les cars de reportage: zone sécurisée destinée au stationnement des cars de reportage des chaînes de télévision.

-Système de haut-parleurs: système électronique de haut-parleurs permettant la diffusion simultanée des messages audio à l'ensemble des secteurs du stade.

\section{1 -Terrain de jeu :}

L'état actuel : Le terrain de football en gazon naturelle, est de 110 mètres de longueur su une largeur 90 mètres. En 2002 le stade été fermé pour une pose de pelouse naturelle, actuellement considérée comme la meilleure en Algérie. Le terrain dispose d'un système de drainage, mais dépourvu d'un dispositif de chauffage. (4)

Le terrain a une incidence directe sur la qualité de la pratique sportive, le choix du sol sportif se fait en fonction du type d'activités, du niveau de pratique et de l'intensité d'utilisation.

\section{Eclairage :}

Durant la phase de conception et de construction d'un stade, il convient de privilégier les technologies permettant de réaliser des économies d'énergie ; l'éclairage électrique est fonction des sources lumineuses dont les qualités requises sont :

-les niveaux d'éclairement.

-les exigences des retransmissions télévisées.

-l'éclairage des gradins.

-l'éclairage de sécurité.

3.1.1 L'état actuel : Le stade dispose de 04 pylônes de 10 mètres de hauteur, chacun dispose 32 lampes pour l'éclairage nocturne, et 20 pylônes pour l'éclairage périphérique.(2)

(Nous n'avons pas pu calculer l'éclairage en unité demandé par le règlement de la FIFA.)

3.1.2 Eclairage de secours :

Pour assurer l'éclairage de secours, le complexe dispose de 03 postes de transformation d'énergie électrique qui permette son alimentation en cas d'une panne générale.

\subsubsection{Zone d'échauffement :}

Etat actuel : La zone d'échauffement est parfaitement soumise aux règlements. (4)

Buts et but de réserve : Les buts de football de dimensions réglementaires $7,32 \times 2,44$ mètres en aluminium blanc, conforme aux règlements de la FIFA. (4)

3.1.4 Banc des remplaçants : conforme aux règlements.

3.1.5 Mâts pour drapeau : conforme aux règlements. (4)

3.2 Vestiaires : 


\section{Dr. SIAF FOUAD}

L'état actuel : Il s'agit de 02 blocs, construits en rez-de-chaussée, avec une aération supérieure.

-Le premier bloc couvre une surface de $: \mathbf{3 8 0} \mathbf{~ m}^{2}$

-Le deuxième bloc couvre une surface de $\mathbf{7 4 0} \mathbf{~ m}^{2}$ (2)

- Ces deux blocs abritent 12 vestiaires pour les joueurs, et 02 vestiaires pour les arbitres.

- Le bloc sanitaire est composé de 11 douches collectives.

3.2.1 Local du délégué: Conforme aux règlements. (4)

3.2.2 Local des premiers secours et de soins pour les joueurs et les Officiels : Le stade est dépourvu d'un local pour les premiers secours, lors des matches les soins des joueurs s'effectuent au niveau de la salle de contrôle antidopage. (4)

3.2.3 Local de contrôle antidopage : Conforme aux règlements. (4)

3.3 Places pour stationnement : le stade dispose d'un parc de stationnement privilégié pour les VIP, bien sécurisé lors des compétitions.

3.4.1 Tribunes et installations pour les spectateurs : Chaque secteur du stade, à savoir et surtout les entrées, les sorties, les escaliers, les portes, les issues de secours, les toits et tous les espaces publics et privés doivent être conformes aux normes de sécurité des autorités locales compétentes.

La capacité des places assises est de 35.000 places, 20.000 places dans la partie non couverte et 15.000 places dans la partie couverte.

Les voies de passage et les escaliers publics dans les espaces accessibles aux spectateurs doivent être clairement indiqués, tout comme les portes menant de ces espaces vers les tribunes et toutes les issues menant hors du stade. L'accès à toutes les voies de passage, couloirs, escaliers et portes publiques doit être constamment dégagé afin de ne pas entraver le flux de spectateurs.

Les gradins reposent directement sur le sol et sont réalisés en béton armé.

-Ils ont une forme trapézoïdale de 15 mètres de largeur et 25 mètres de longueur, protégés par un mur en béton de 1 mètre de hauteur du coté amont.

-Ils sont séparés entre eux, tous les 3 mètres, par un joint qui joue le rôle de drain pour les eaux pluviales.

-Des escaliers sont implantés tous les 12 mètres.

-Les gradins ne disposent pas de sièges individuels.

-Des point de vente de boissons et de restauration rapides sont ouverts aux spectateurs à proximité des gradins, au nombre de 06 buvettes en béton de $\mathbf{1 0 \mathbf { m } ^ { 2 }}$.

-Les gradins sont séparés en secteur indiqué par des plaques signalétiques numérotées. (2)

3.4.2 Entrées et sorties réservées au public : Les portes de sortie et d'entrée du stade et les portes menant des espaces des spectateurs aux tribunes doivent s'ouvrir vers l'extérieur du point de vue des spectateurs. Elles ne doivent jamais être verrouillées tant que des spectateurs se trouvent dans le stade.

-Le stade disposant de 3 abords :

-Le premier pour les officiels qui a trois grands portails.

-Le deuxièmes pour les spectateurs sur le coté des tribunes. 
- Le troisième pour les spectateurs qui dispose de six grands portails et six portes.

-Ces accès ne disposent pas d'indication explicite depuis les entrés de l'agglomération.

-Les portails ne sont pas clairement indiqués par de dispositifs de signalisation ni de système de contrôle d'accès. (2)

3.4.3 Installations sanitaires pour les spectateurs : le stade dispose de 08 toilettes pour public sous sol autour du stade.

3.5.1 Locaux de premiers secours pour les spectateurs : Le stade est dépourvue des locaux de premier secours, les soins s'effectuent à une zone située sous le pont marathon ou s'installent les ambulances. (2)

3.5.2 Installations pour les spectateurs handicapés : Le stade ne dispose d'aucun des critères exigés par le règlement de la FIFA pour les spectateurs handicapés. (2,et 4 ) Places VIP : Le stade dispose d'une tribune officielle protégée en plexiglas de 200 places. L'utilisation de l'espace VIP, source indispensable de revenus, est également optimisée.

\subsubsection{Zones réservées aux médias :}

\section{Zone de travail des médias :}

-Au moins une salle équipée de pupitres, de prises électriques et de prises de téléphone/connexions Internet doit être mise à la disposition des représentants des médias.

\section{L'état actuel :}

-02 cabines de presse de $16 \mathrm{~m}^{2}$.

Positions des caméras : Une plate-forme pour la caméra principale doit être mise à disposition dans la tribune principale. Elle doit être située au centre de la tribune, suffisamment élevée par rapport au terrain pour garantir une qualité d'image optimale.

L'emplacement de la caméra principale doit se trouver exactement dans le prolongement de la ligne médiane, à une hauteur correspondant à un angle de 15-20 avec le plan horizontal depuis le rond central. La plate-forme pour la caméra principale doit mesurer $6 \mathrm{~m}^{2}$ au minimum, afin de pouvoir accueillir deux caméras.

L'état actuel : -Non conforme aux règlements. (4)

Tribune de presse : La tribune de presse doit être couverte et occupe une position centrale dans la tribune principale. Elle doit offrir une vue dégagée sur l'ensemble de la zone de jeu et un accès facile aux autres zones des médias. Dans la tribune de presse, toutes les places assises avec pupitre doivent être munies d'une prise électrique et de prises de téléphone/connexions Internet. Les pupitres doivent être suffisamment grands pour y placer un ordinateur portable et un bloc-notes.

L'état actuel :

- La tribune de presse n'est pas séparée de celle du média. (4)

- Elle ne dispose pas de critères cités dans les règlements de la FIFA. (4)

\subsection{Budget réservé au stade avant les travaux de réhabilitations en 2007}




\section{Dr. SIAF FOUAD}

Un Budget réservé au stade avant les travaux de réhabilitations en 2007 estimé à 540.120 .000 de dinars, une autre enveloppe financière estimée 150.200.000 de dinars qui été mobilisée pour les travaux supplémentaires (travaux retenus dans le cadre de la réhabilitation) en 2007. (1)

Tableau $\mathrm{N}^{\circ} 01$ : Estimation du cout du stade avant les travaux de réhabilitations en 2007 :

\begin{tabular}{|l|l|c|}
\hline $\mathrm{N}^{\circ}$ & DESIGNATION DES OUVRAGES & Montant/en Dinars \\
\hline 01 & Infrastructure & 132.000 .000 \\
\hline 02 & Pistes de circulation & 13.200 .000 \\
\hline 03 & Structure $\quad \rightarrow$ Tribune & 86.400 .000 \\
& \multicolumn{1}{|c}{ Gradins } & 144.000 .000 \\
\hline 04 & Pelouse et système de drainage & 70.000 .000 \\
\hline 05 & Piste a 08 couloires & 30.000 .000 \\
\hline 06 & Eclairage+Tableau d'affichage & 10.000 .000 \\
\hline 07 & Blocs Administratif+ Vestiaires +Sanitaire & 20.000 .000 \\
\hline 08 & Espaces verts et parkings & 20.000 .000 \\
\hline 09 & Stade réplique & 13.200 .000 \\
\hline 10 & Voies et réseaux divers & 1.320 .000 \\
\hline & TOTALE GENERAL & 540.120 .000 \\
\hline
\end{tabular}

Tableau $\mathrm{N}^{\circ} 02$ : Travaux supplémentaires (travaux retenus dans le cadre de la réhabilitation)

\begin{tabular}{|l|l|c|}
\hline $\mathrm{N}^{\circ}$ & DESGNATION DES OUVRAGES & Montant/en Dinars \\
\hline 01 & Mur de clôture & 55.000 .000 \\
\hline 02 & Vestiaires & 15.000 .000 \\
\hline 03 & Administration & 6.000 .000 \\
\hline 04 & Depot & 2.000 .000 \\
\hline 05 & Poste de transformation (électricité) & 1.500 .000 \\
\hline 06 & Tribunes officielles & 30.000 .000 \\
\hline 07 & Réseau adduction eau potable +incendie & 4.700 .000 \\
\hline 08 & Eclairage périphérique extérieure & 3.000 .000 \\
\hline 09 & Réseau d'assinissement & 30.500 .000 \\
\hline 10 & Logement de fonction & 2.500 .000 \\
\hline & TOTALE GENERAL & 150.200 .000 \\
\hline
\end{tabular}

-Rapport d'expertise 2006, réalisé par Larradj Ali expert industriel (1)

TOTAL GENERAL 690.320.000. DA représente les travaux de réhabilitation.

Ces travaux de rénovation ou de réaménagement coûtent souvent plus cher qu'une nouvelle construction, celle-ci permettant également de disposer d'une infrastructure plus moderne. 


\section{CONCLUSION :}

Nous avons constaté que le stade est dépourvu de certains critères techniques, ce qui peut affecter le confort et la sécurité des spectateurs, surtout lors de leur afflux au niveau des abords. Pour cette raison il est recommandé de :

-la mise en place d'un Poste Médical Avancé (PMA) ou des postes de secours.

-La mise en place des sièges individuels inflammable, incassable avec dossier de 30 $\mathrm{cm}$, sur les contremarches des gradins, favorisant l'obtention d'un épuré de visibilité satisfaisante et facilite la surveillance et le nettoyage.

-Elimination complète des places debout pour des raisons de confort et sécurité, en effet rester debout pour des heures peut générer des facteurs de fatigue et d'énervements ce qui peut induit l'agressivité des spectateurs.

-Mise en place des sièges spécifiques pour les fauteuils roulants.

-Intégration d'un système de sonorisation qui permet de renseigner les spectateurs sur le déroulement des matchs, encore pour diffuser des messages publicitaires, et en cas de besoin de passer des informations spécifiques.

-Mettre en place les équipes compétentes pour exploiter les stades,

-La surface de jeu en gazon naturel offre aux footballeurs des conditions qui favorisent un développement du football notamment sur l'aspect technique, tactique, sécuritaires, une meilleure rentabilité et un spectacle de qualité ;

-Le complexe sportif permettra à Constantine de postuler à l'accueil des manifestations sportives de haut niveau; ainsi la promotion du sport d'élite notamment le football.

- Selon l'auteur G.Tribou 2004 (05) «Le club professionnel de football sportif est désormais une entreprise de type éducatif, et le dirigeant responsable doit être un éducateur doublé d'un bon gestionnaire (...) capable de fixer des objectifs et de savoir les confronter aux moyens» .

La qualité de ce stade doit être exploité par le club professionnel de Constantine (CSC) notamment pour les recettes additionnelles (billetterie, loges,)

\section{BIBLIOGRAPHIE :}

1-Rapport d'expertise 2006, réalisé par Larradj Ali expert industriel et membre titulaire de l'ORDINEX.

2-Rapport d'expertise et étude des risques complexe OPOW et ses annexes.

3-Texte de référence : Le journal officiel :

- Arrêté $\mathrm{N}^{\circ} 70.038 \mathrm{du}$ 05-05-1970.

4-Décret exécutif n ${ }^{\circ}$ 09-184 du 17 Joumada El Oula 1430 correspondant au 12 mai 2009 fixant les procédures, et les normes spécifiques de l'homologation technique et sécuritaire des infrastructures sportives ouvertes au public ainsi que les modalités de leur application.

05-Tribou G. Sponsoring sportif, édition Economica, Paris. 2004. 